10,19

\title{
О статистическом распределении в деформируемом твердом теле
}

\author{
() Н.Н. Горобей, А.С. Лукьяненко \\ Санкт-Петербургский политехнический университет Петра Великого, \\ Санкт-Петербург, Россия \\ E-mail: n.gorobey@mail.ru
}

(Поступила в Редакцию 25 января 2017 г.)

Предложена модификация распределения Гиббса для теплоизолированного механически деформируемого твердого тела, в которой его линейные размеры (параметры формы) исключены из статистического усреднения и включены, наряду с температурой, в состав макроскопических параметров состояния. Формально модификация сводится к соответствующим дополнительным условиям при вычислении статистической суммы. Сами параметры формы и температура находятся из условий механического и термодинамического равновесия тела, а их изменение - с использованием первого начала термодинамики. Для простой модели твердого тела - ансамбля ангармонических осцилляторов в рамках предложенного формализма с точностью до первого порядка по константе ангармонизма анализируются известные термо-механические явления. Модификация распределения рассмотрена отдельно для классической и квантовой областей температур.

DOI: $10.21883 /$ FTT.2017.09.44853.017

\section{1. Введение}

Основой статистической механики является распределение Гиббса, которое необходимо для вычисления среднего значения любой наблюдаемой в системе, находящейся в термодинамическом равновесии с тепловым резервуаром (термостатом). Как отмечается в [1], это распределение годится и для замкнутых тел, исключая лишь рассмотрение вопроса о флуктуациях полной энергии тела, которая для замкнутых тел по определению постоянна. Однако есть еще одна задача, в которой использование распределения Гиббса для замкнутого тела потребует некоторой модификации. Речь идет о так называемом термо-упругом (ТУ) эффекте [2,3] зависимости температуры теплоизолированного твердого тела от внешней механической нагрузки, который является проявлением нелинейности сил межатомного взаимодействия (ангармонизма). Сам факт этой зависимости, при внимательном рассмотрении, указывает на необходимость модификации распределения Гиббса, в частности, при вычислении внутренней энергии адиабатически деформируемого твердого тела. Термодинамика ТУ-эффекта рассмотрена в работах [4-6]. На примере одного осциллятора он объясняется параметрической зависимостью энергии колебаний от деформации за счет ангармонического „смягчения“ постоянной упругости осциллятора [7]. Еще одним проявлением ангармонизма является термическое расширение твердого тела, которое может быть вычислено с использованием распределения Гиббса для атомных колебаний без всяких модификаций. Например, в [8] оно находится для простейшей модели ангармонического твердого тела как среднее смещение из положения равновесия колеблющегося ангармонического осциллятора. В то же время, зависимость от нагрузки средней энергии колебаний того же осциллятора, вычисленной с использованием обычного распределения Гиббса, как мы увидим, с достаточной точностью (первый порядок теории возмущений по константе ангармонизма) отсутствует. Это означает, что для объяснения ТУ-эффекта статистическое распределение следует модифицировать. Предлагаемая в данной работе модификация распределения Гиббса сводится к тому, что в изолированном твердом теле часть динамических переменных, характеризующих линейные размеры тела, исключается из состава микроскопических, подлежащих статистическому усреднению. Наряду с температурой, они образуют набор параметров состояния тела, определяемых внешними условиями, в частности, условиями механического равновесия. В системе с большим числом частиц всегда могут быть найдены такие динамические переменные, относительные флуктуации которых пренебрежимо малы. Рассматривая в качестве модели ангармонического твердого тела большой ансамбль ангармонических осцилляторов, естественно считать таким классическим параметром состояния ансамбля среднее (по ансамблю) смещение осциллятора из положения равновесия

$$
\varepsilon=\frac{1}{N} \sum_{n=1}^{N} x_{n},
$$

где $x_{n}$ - мгновенные значения координат осцилляторов. Возможность пренебречь флуктуациями этой величины в случае большого ансамбля основана на законе больших чисел и не зависит от выбора статистики, включая и квантовую. Таким образом, для большого ансамбля эта величина „выбывает“ из статистического усреднения, а ее значение, как мы увидим, определяются из условия механического равновесия. В данной работе на примере ансамбля ангармонических осцилляторов показано, что соответствующая модификация распределения Гиббса позволяет учесть влияние внешней механической нагрузки на среднюю энергию их колебаний и, тем самым, объяснить ТУ-эффект с любой требуемой точностью. 


\section{2. Статистическая сумма ансамбля ангармонических осцилляторов (высокие температуры)}

Внутренняя энергия ансамбля одномерных ангармонических осцилляторов при высоких температурах может быть найдена на основе распределения Гиббса после вычисления классической статистической суммы

$$
Z \equiv \iint d x d p \exp [-\beta H(x, p)]
$$

где $\beta=1 / k T$ и

$$
H(x, p)=\frac{p^{2}}{2 m}+U(x)-F x
$$

— гамильтониан осциллятора, куда мы включили потенциальную энергию внешнего силового поля. Мы ограничимся здесь приближением кубического ангармонизма

$$
U(x)=\frac{f x^{2}}{2}-\frac{g x^{3}}{3}
$$

Для получения конечной статистической суммы, интеграл (2) следует вычислять разложением экспоненты в ряд по степеням константы ангармонизма $g$. Нетрудно видеть, что с точностью до первого порядка малости по $g$ средняя энергия осциллятора равна

$$
w \equiv-\frac{d \ln Z}{d \beta}=k T-\frac{F^{2}}{2 f}-\frac{g}{3}\left(\frac{F}{f}\right)^{3},
$$

где квадратичная относительно упругой деформации часть потенциальной энергии имеет противоположный знак из-за сдвига начала отсчета энергии. Так или иначе, в указанном приближении средняя энергия колебаний осциллятора равна $k T$ и не зависит от приложенной нагрузки. Нас может удовлетворить этот результат, если понимать под температурой $T$ в распределении Гиббса температуру теплового резервуара, с которым взаимодействуют осцилляторы.

Поскольку ТУ-эффект и явление термического расширения являются эффектами первого порядка малости по константе ангармонизма $g$, для учета влияния механической нагрузки на среднюю энергию колебаний осциллятора потребуется модификация распределения Гиббса, следствием которой будет и изменение в понимании параметра температуры распределения. Изолируя осцилляторы от теплового резервуара и допуская лишь слабый обмен энергией между ними в ансамбле, мы приходим к понятию температуры ансамбля. Влияние ангармонизма проявится в том, что температура ансамбля будет отличаться от температуры теплового резервуара на величину, пропорциональную потенциальной энергии среднего смещения осциллятора из положения равновесия.

Предлагаемая модификация распределения заключается в учете соотношения (1) как дополнительного условия в $N$-мерном конфигурационном пространстве ансамбля. В определении статистической суммы мы учтем это дополнительное условие интегрированием по добавочной переменной $\lambda$ в статистической сумме:

$$
\begin{aligned}
\widetilde{Z}_{N}= & \int d \lambda \int \prod d^{N} p d^{N} x \\
& \times \exp \left[-\beta \sum_{n=1}^{N} H\left(x_{n}, p_{n}\right)+i \lambda\left(\sum_{n=1}^{N} x_{n}-N \varepsilon\right)\right],
\end{aligned}
$$

которое дает соответствующую условию (1) $\delta$-функцию. Заметим, что если провести в (6) интегрирование по $\varepsilon$, получим $\delta(\lambda)$, что снимает дополнительное интегрирование по $\lambda$ в (6) и возвращает модифицированную статистическую сумму к исходной (2). Вычисление (6) с требуемой точностью дает

$$
\widetilde{Z}_{N} \approx(2 \pi)^{N}\left(\frac{m}{f}\right)^{N / 2}\left(\frac{2 \pi f}{N}\right)^{1 / 2} \beta^{-(2 N-1) / 2} \exp [-N \beta \widetilde{U}(\varepsilon)],
$$

где

$$
\widetilde{U}(\varepsilon) \equiv U(\varepsilon)-F \varepsilon-\frac{g k T \varepsilon}{f} .
$$

Упомянутый интеграл по $\varepsilon$ с достаточной точностью может быть вычислен с использованием метода перевала (большой параметр $N$ в показателе экспоненты) [9]. В этом приближении, если подставить в (7) значение $\varepsilon$, удовлетворяющее условию экстремума показателя экспоненты, модифицированная статистическая сумма $\widetilde{Z}_{N}$ с достаточной точностью (первый порядок малости по $g$ ) совпадет с исходной, и возможность объяснения ТУ-эффекта опять утрачивается. Само условие экстремума совпадает с условием механического равновесия

$$
f \varepsilon-g \varepsilon^{2}=F+\frac{g}{f} k T,
$$

в котором, помимо внешней силы, присутствует так называемое „тепловое давление“, обусловленное ангармонизмом [10]. Уравнение (6) будет использовано далее, как уравнение состояния ансамбля осцилляторов для определения его температуры и среднего удлинения осциллятора при заданных внешних условиях. Еще одно уравнение, необходимое для этого, получим стандартным образом из статистической суммы (7) в виде выражения для внутренней энергии ансамбля при заданном среднем удлинении осциллятора $\varepsilon$. В расчете на один осциллятор с достаточной точностью эта энергия равна сумме энергии колебаний и потенциальной энергии, связанной со средним удлинением $\varepsilon$,

$$
w \approx k T+U(\varepsilon)
$$

Численное значение энергии $w$ определяется температурой теплового резервуара, с которым контактировали осцилляторы до тепловой изоляции ансамбля, так что мы будем относить величины $F, w$ к внешним параметрам состояния ансамбля. Обратим внимание, что часть 
энергии, полученной от теплового резервуара ансамблем, „резервируется“ в виде потенциальной энергии деформации $\varepsilon$, которая в данной осцилляторной модели соответствует квазистатической потенциальной энергии ангармонического твердого тела, введенной в [11]. Поэтому температура ансамбля ангармонических осцилляторов $T$ несколько ниже (при $g>0$ ) температуры теплового резервуара, от которого получена энергия $w$. Тот факт, что внутренняя энергия (10) вычисляется для модифицированной статистической суммы (7) при фиксированном среднем смещении осциллятора $\varepsilon$ означает, что мы имеем дело с распределением Гиббса для теплоизолированного ансамбля. Действительно, в контакте с термостатом энергия колебаний каждого осциллятора, а вместе с этим и среднее смещение из положения равновесия $\varepsilon$, испытывали бы значительные флуктуации.

Систему уравнений (9) и (10) следует решать совместно. В результате мы получаем параметры состояния ансамбля $(\varepsilon, T)$ как функции внешних параметров $(F, w)$. Так, уравнение (9) определяет с достаточной точностью среднее смещение из положения равновесия осциллятора $\varepsilon$ как сумму механической деформации и термического расширения

$$
\varepsilon \approx \frac{F}{f}+\frac{g}{f^{2}} k T .
$$

Если в начальном состоянии ансамбля внешняя нагрузка отсутствует $(F=0)$, деформация сводится к термическому расширению

$$
\varepsilon_{0} \approx \frac{g}{f^{2}} k T_{0}
$$

Теперь уравнение (10) позволяет найти изменение температуры ансамбля за счет добавочной механической деформации,

$$
\Delta \varepsilon \approx \frac{F}{f},
$$

с учетом того, что в условиях адиабатического нагружения изменение внутренней энергии ансамбля равно работе внешней силы на деформации (13),

$$
\Delta w \approx \frac{F^{2}}{2 f} .
$$

Это изменение с достаточной точностью равно

$$
\Delta T \approx-T_{0} \frac{g T}{f^{2}}
$$

что и объясняет классический ТУ-эффект.

\section{3. Статистическая сумма ансамбля ангармонических осцилляторов. Низкие температуры}

В области низких температур, где существенны квантовые эффекты, обычная статистическая сумма вычисля- ется по формуле [1]

$$
Z \equiv \operatorname{Tr} e^{-\hat{H}}
$$

где

$$
\widehat{H} \equiv-\frac{\hbar^{2}}{2 m} \frac{d^{2}}{d x^{2}}+U(x)-F x
$$

- оператор Гамильтона осциллятора во внешнем силовом поле. Предлагаемая здесь модификация распределения Гиббса для изолированного ансамбля осцилляторов также сводится к введению, наравне с температурой, классического параметра состояния - среднего смещения из положения равновесия $\varepsilon$. Соответствующая модифицированная статистическая сумма ансамбля имеет вид

$$
\widetilde{Z}_{N}=\int d \lambda \operatorname{Tr} \exp \left[-\beta N \widehat{H}+i \lambda\left(\sum_{n=1}^{N} x_{n}-N \varepsilon\right)\right] .
$$

Найдем сначала с достаточной точностью (до первого порядка по константе ангармонизма $g$ включительно) модифицированную статистическую сумму для одного квантового ангармонического осциллятора

$$
\widetilde{Z} \equiv \operatorname{Tr} \exp (-\beta \widehat{H}+i \lambda x) .
$$

Здесь, как и в классическом случае, раскладываем операторную экспоненту в ряд по степеням $g$, а след вычисляем с использованием базиса собственных функций невозмущенного гармонического осциллятора. При этом, чтобы не нарушать эрмитовость модифицированного гамильтониана, введем на данном этапе вещественный параметр $\Lambda: i \lambda \rightarrow \Lambda$, а перед интегрированием по $\lambda$ сделаем обратное преобразование. Вычисление дает

$$
\widetilde{Z} \approx e^{\frac{\Lambda^{2}}{2 \beta f}}\left[\left(1+\frac{g \Lambda^{3}}{3 \beta^{2} f^{3}}\right) Z_{0}+\frac{g \Lambda}{f^{2}}\langle w\rangle_{0}\right],
$$

где

$$
Z_{0} \equiv \sum_{k=0}^{\infty} \exp \left[-\beta \hbar \omega\left(k+\frac{1}{2}\right)\right]
$$

- обычная статистическая сумма для гармонического осциллятора с собственной частотой $\omega=\sqrt{f / m}$ и

$$
\langle w\rangle_{0}=-\frac{d \ln Z_{0}}{d \beta}
$$

его средняя энергия. Подставляя (20) в (18), после обратной замены $\Lambda \rightarrow i \lambda$ вычисляем с достаточной точностью интеграл по $\lambda$

$$
\widetilde{Z}_{N} \approx Z_{0}^{N} \exp N\left[-\beta U(\varepsilon)-\beta F \varepsilon+\beta \frac{g \varepsilon}{f^{2}}\langle w\rangle_{0}\right] .
$$

Как и при высоких температурах, имеем систему двух уравнений для параметров состояния $(\varepsilon, T)$. Это - 
уравнение состояния, которое вытекает из условия экстремума показателя экспоненты

$$
f \varepsilon-g \varepsilon^{2}=F+\frac{g}{f^{2}}\langle w\rangle_{0}
$$

и уравнение для внутренней энергии ансамбля (в расчете на один осциллятор), которое получается обычным образом из модифицированной статистической суммы (18)

$$
\langle w\rangle_{0}+U(\varepsilon)-\frac{g \varepsilon}{f^{2}} \frac{\partial}{\partial \beta}\left(\beta\langle w\rangle_{0}\right) \approx w
$$

При высоких температурах $\left(\langle w\rangle_{0} \approx k T\right)$ уравнения (24) и (25) сводятся к (9) и (10) соответственно. При низких температурах $\left(\langle w\rangle_{0} \approx \hbar \omega / 2\right)$ уравнение (24) определяет среднее смещение $\varepsilon$ как сумму механической деформации и удлинения, связанного с нулевыми колебаниями осцилляторов. Уравнение (25) тогда определяет зависимость температуры ансамбля от механической деформации. В рассматриваемом пределе низких температур, с учетом третьего слагаемого в левой части уравнения (25), эта зависимость, т.е. ТУ-эффект, отсутствует. Полученный результат согласуется с выводами работы [12]. Подчеркнем, что здесь он получен за счет модификации распределения Гиббса, основанной на введении классического параметра состояния ансамбля - среднего смещения из положения равновесия осциллятора $\varepsilon$.

\section{4. Заключение}

Таким образом, модификация распределения Гиббса для замкнутого тела, в которой исключается статистическое усреднение по макроскопическим параметрам состояния тела, таким, как его линейные размеры (в осцилляторной модели - среднее удлинение осциллятора), позволяет получить зависимость его температуры от механической деформации в условиях адиабатичесого нагружения. Рассматривая линейные размеры тела, наряду с температурой, в качестве параметров состояния изолированного тела, получаем для них замкнутую систему уравнений, определяющую их зависимость от внешних условий. Часть этих уравнений имеет физический смысл условий механического равновесия тела во внешнем силовом поле (уравнения состояния), которые определяют деформации его линейных размеров. Дополнительным к этим уравнениям состояния получено выражение для внутренней энергии тела, в котором явно учитывается потенциальная энергия деформаций как следствие модификации распределения Гиббса. С учетом закона сохранения энергии в процессе адиабатического нагружения (первое начало термодинамики), изменение температуры тела является следствием изменения потенциальной энергии деформаций.

Авторы благодарят А.В. Гольцева за обсуждение результатов работы.

\section{Список литературы}

[1] Л.Д. Ландау, Е.М. Лифшиц. Статистическая физика. Наука, М. (1976). Ч. 1. 583 c.

[2] J.P. Joule. Proc. R. Soc. 8, 564 (1857).

[3] W. Thompson (Lord Kelvin). Trans. Roy. Soc. Edinburgh 20, 261 (1853).

[4] R.T. Potter, L.J. Greeves. Proc. SPIE 817, 134 (1987).

[5] A.A. Benam, G. Viola, T. Korakianitis. J. Therm. Anal. Calorim. 100, 941 (2010).

[6] В.Л. Гиляров, А.И. Слуцкер, В.П. Володин, А.И. Лайус. ФТT 40, 1548 (1998).

[7] A.I. Slutsker, V.P. Volodin. Thermochimia Acta 247, 111 (1994).

[8] Ч. Киттель. Введение в физику твердого тела. Гостехиздат, М. (1957). 524 c.

[9] Н.Н. Горобей, А.С. Лукьяненко. ФТТ 56, 2187 (2014).

[10] В.Р. Регель, А.И. Слуцкер, Э.Е. Томашевский. Кинетическая природа прочности твердых тел. Наука, М. (1974). $560 \mathrm{c}$.

[11] А.И. Слуцкер, Ю.И. Поликарпов, Д.Д. Каров, И.В. Гофман. ФТT 55, 610 (2013).

[12] В.Л. Гиляров, А.И. Слуцкер. ФТТ 56, 2407 (2014). 